\title{
When Does Haste Make Waste? Speed-Accuracy Tradeoff, Skill Level, and the Tools of the Trade
}

\author{
Sian L. Beilock \\ University of Chicago \\ Michael Hoerger \\ Central Michigan University
}

\author{
Bennett I. Bertenthal \\ Indiana University \\ Thomas H. Carr \\ Vanderbilt University
}

\begin{abstract}
Novice and skilled golfers took a series of golf putts with a standard putter (Exp. 1) or a distorted funny putter (consisting of an s-shaped and arbitrarily weighted putter shaft; Exp. 2) under instructions to either (a) take as much time as needed to be accurate or to (b) putt as fast as possible while still being accurate. Planning and movement time were measured for each putt. In both experiments, novices produced the typical speed-accuracy trade-off. Going slower, in terms of both the planning and movement components of execution, improved performance. In contrast, skilled golfers benefited from reduced performance time when using the standard putter in Exp. 1 and, specifically, taking less time to plan improved performance. In Exp. 2, skilled golfers improved by going slower when using the funny putter, but only when it was unfamiliar. Thus, skilled performance benefits from speed instructions when wielding highly familiar tools (i.e., the standard putter) is harmed when using new tools (i.e., the funny putter), and benefits again by speed instructions as the new tool becomes familiar. Planning time absorbs these changes.
\end{abstract}

Keywords: expertise, speed-accuracy trade-off, skill acquisition, automaticity

From the early skill acquisition research of Bryan and Harter (1899) to the present day, investigators of human skill learning have explored differences in expert and novice execution in an attempt to shed light on the variables that mediate optimal performance. Successful investigations of such learning move beyond merely cataloguing overt task performance, instead identifying changes in the cognitive control structures that underlie execution at various stages along the skill acquisition continuum (Anderson, 1993; Kanfer \& Ackerman, 1989; Proctor \& Dutta, 1995). Not only does such work contribute to a better understanding of the cognitive substrate governing optimal performance, but it also provides researchers and practitioners alike with methods for differentiating those most skilled from their less skilled counterparts.

Cognitive theories of skill acquisition and automaticity suggest that novel skill performance is based on explicitly retrievable declarative knowledge that is held in working memory and consciously attended in real time (Anderson, 1983, 1993; Fitts \&

Sian L. Beilock, Department of Psychology, University of Chicago; Bennett I. Bertenthal, Department of Psychological and Brain Sciences, Indiana University; Michael Hoerger, Department of Psychology, Central Michigan University; and Thomas H. Carr, Psychology and Human Development, Vanderbilt University.

Carr is now in the Department of Psychology, Michigan State University.

This work is supported by NSF Grants BCS-0601148 to S. L. Beilock and SBE9704764 to B. I. Bertenthal.

Correspondence concerning this article should be addressed to Sian L. Beilock, Department of Psychology, 5848 South University Avenue, University of Chicago, Chicago, IL 60637. E-mail: beilock@uchicago.edu
Posner, 1967; Proctor \& Dutta, 1995). As learning progresses, information is believed to be restructured into proceduralized performance processes that operate largely outside of working memory (Anderson, 1983, 1993; Brown \& Carr, 1989; Fitts \& Posner, 1967; Keele, 1986; Keele \& Summers, 1976). Proceduralized skill representations do not mandate the same degree of attention and control that was necessary at lower levels of practice and are supported by different neural structures than were active early in learning (Chein \& Schneider, 2005; Jancke, Shah, \& Peters, 2000; Milton, Solodkin, Hlustik, \& Small, 2007; Raichle et al., 1994).

The notion that different cognitive processes underlie various stages of skill acquisition-with a trend toward increased proceduralization at higher levels of expertise-carries implications for the types of attentional manipulations that may influence performance across levels of learning. For example, it has been demonstrated that skilled performers operating in familiar environments in tasks ranging from golf putting to soccer dribbling to baseball batting benefit from conditions that limit attention to execution (e.g., attention-demanding secondary tasks) in comparison to conditions that prompt step-by-step control (e.g., focusing on a specific mechanical aspect of execution), whereas novices show the opposite pattern (Beilock, Carr, MacMahon, \& Starkes, 2002; Castaneda \& Gray, 2007; Gray, 2004; Jackson, Ashford, \& Norsworthy, 2006; Ford, Hodges, \& Williams, 2005; Perkins-Ceccato, Passmore, \& Lee, 2003). Because novice execution demands attentional resources that skilled performance does not, conditions that direct attention away from primary task execution hurt novices more than skilled performers. In contrast, conditions that direct attention to the unfolding of performance hurt skilled performers 
more than novices (Beilock, Jellison, Rydell, McConnell, \& Carr, 2006).

Furthermore, such effects are not limited to situations that explicitly direct performers' attention to specific task goals or demands. Merely varying the time available for execution differentially impacts performance as a function of skill level. Beilock, Bertenthal, McCoy, and Carr (2004) had novice and skilled golfers take a series of ordinary 4 and 5 foot putts on an indoor putting green under instructions emphasizing putting quickly or instructions prompting individuals to take as much time as needed to be accurate. Skilled golfers were more accurate when prompted to putt faster than when given unlimited execution time. Novices showed the opposite pattern-performing more accurately when given more rather than less execution time. Because controlled attention takes time to deploy (Posner \& Snyder, 1975; Shiffrin \& Schneider, 1977), situations that limit the deployment of attention during the planning, selection, and run-off of a skilled action should hurt novice performance that requires such resources. In contrast, these same time constraints may actually benefit skilled performance if they prevent individuals from explicitly trying to control aspects of execution better left outside conscious awareness.

Admittedly, the idea that performance might benefit from speed instructions goes against the well-established speed-accuracy trade-off (Fitts, 1954; Woodworth, 1899)—which has been shown to extend to both the preparation and movement components of a skill (MacKay, 1982). It should be noted, however, that a majority of speed-accuracy work to date has been conducted with individuals performing novel or relatively unfamiliar skills (Schmidt \& Lee, 2005). This certainly does not hold for the skilled golfers tested by Beilock, Bertenthal, McCoy, \& Carr (2004). In fact, if one turns to recent work examining the impact of speed instructions on performance, the notion that the more time spent preparing and executing a skill, the better the outcome does not always holdespecially for those with experience performing the task at hand.

Johnson and Raab (2003) examined the time course of actionbased decision making in team handball players - a game in which teams of six players plus a goalie try to throw a ball into the opposing team's goal. Individuals watched videos of high-level games. The videos were paused at certain points and participants were instructed to imagine that they were the player who currently possessed the ball and to select the best possible action to perform next (e.g., where to pass the ball) as quickly as possible. The screen then remained frozen for $45 \mathrm{~s}$, and the players indicated all other possible actions they (as the player with the ball) might perform. Players were then asked to determine the overall best possible action. This final judgment differed from the initially named action about $40 \%$ of the time. From a speed-accuracy trade-off standpoint, one might predict the final judgment would be a better decision than the initial one-after all, players had more time to evaluate the former as compared to the latter decision. However, this was not the case. Overall, the first actions selected by the players were better decisions than the final ones (as determined by professional handball coaches). To the extent that actions are selected on the basis of strength of association to an environmental stimulus, and better action options have stronger connections for experienced players operating in familiar environments (Klein et al., 1995), action selection may benefit from time constraints. And indeed, in a follow-up study explicitly exploring action selection as a function of handball skill level, Raab and
Johnson (2007) found that handball expertise increased the quality of initially generated action options as well as the speed with which such options came to mind. Thus, whether individuals are merely choosing an action (as in Johnson \& Raab, 2003), or choosing and executing an action (as in Beilock, Bertenthal, McCoy, \& Carr, 2004), time constraints appear to aid performance to the extent that performers have some skill and experience with the task in question. In certain situations, haste may help.

But what happens when the constraints of performance change? Suppose that skilled performers are forced to plan, fine-tune, or execute actions in unfamiliar environments or suppose that athletes are given a new tool with which to implement their skillespecially a redesigned tool with a new or unusual configuration (e.g., a baseball bat made out of a new and heavier material, or a new type of golf club)? If wielding a new tool requires a return to explicit skill planning and control, conditions that limit the deployment of attention should harm skilled performers-just as such conditions harm novices. Such a finding would not only demonstrate the dynamic and changing nature of skilled performance (Gray, 2004), it would also suggest that the impact of speed instructions on performance might serve as a diagnostic tool for assessing skill level when performing in changing environments or with an unfamiliar tool. That is, the extent to which speed instructions alter performance, and in what direction-whether positively or negatively-could serve as an additional and perhaps quite sensitive measure of level of adaptation to a particular performance environment or tool.

\section{Current Experiments}

The current work examined the impact of time constraints on performance as a function of skill level and as a function of participants' familiarity with the tools available to execute their skill. In Experiment 1, novice and skilled golfers took a series of golf putts on an indoor putting green using a standard putter and ball under instructions to either putt as fast as possible (while still being accurate) or to take as much time as needed to optimize accuracy. This first experiment replicated Beilock, Bertenthal, McCoy, \& Carr (2004) and advanced this previous work by examining where in the real-time unfolding of performance speed instructions exert their impact (e.g., preparation vs. movement components of execution). Novice and skilled golfers were videotaped and the time spent in the preparation and movement components of execution were correlated with putting outcome. Understanding which component (or components) of skill execution are most strongly modulated by speed instructions and how the timing of individual putting elements relate to overall putting outcomes will not only shed light on differences in novice and skilled performance, but may also elucidate how-within a given skill level - the control of a particular performance changes as it unfolds in real time.

Regardless of where in the skill execution process the effect of speed instructions occur, if such time constraints do have systematically different effects on performance as a function of skill level-with less skilled performance suffering from time limitations and more skilled performance benefiting-a specific follow-up prediction can be made. Namely, changing the familiarity of the performance environment such that highly skilled individuals are now forced to attend to the novel constraints of 
execution, much like a novice, should result in a reverse in the impact of time constraints on execution-at least until skilled performers have adapted to the new constraints of the task.

In Experiment 2, we altered the tools of our golfers' trade to address this familiarity issue. Novice and skilled golfers again performed a golf putting task under instructions that either emphasized putting fast or instructions in which no time constraints were imposed. However, rather than using a normal putter, all participants used a funny putter consisting of a regular putter head attached to an "s" shaped and arbitrarily weighted putter shaft (Beilock \& Carr, 2001). The design of the funny putter was intended to require experienced golfers to alter well-practiced putting to compensate for the distorted club, forcing them to allocate attention to the new execution processes (Beilock, Wierenga, \& Carr, 2002). To the extent that attention takes time to deploy, and skilled golfers using the funny putter do not possess a well-established action sequence for wielding this novel tool, then such individuals should be hurt by conditions that limit performance time. Novices should not be differentially impacted by the funny putter in comparison to a regular putter. After all, to a novice, all putters should be funny.

\section{Experiment 1}

Novice and skilled golfers took a series of golf putts on our indoor putting green under conditions prompting faster performance (speed instructions) or under conditions in which no time constraints were imposed (accuracy instructions). All participants were videotaped so that their performance could later be digitized and analyzed on a frame-by-frame basis to obtain an accurate measurement of the components of putting time.

If putting faster affords less time for the planning, selection, and control of execution, then time constraints should have opposite effects on novice and skilled performance. As described above, novice (but not skilled) performance is thought to demand the explicit allocation of attention to the planning and implementation of skill processes and procedures. Thus, conditions that limit the deployment of attention should harm the novice performers but not skilled performers. Where in the putting process such time constraints exert their impact however-planning versus movement execution-is an open question.

\section{Method}

\section{Participants}

Novices $(n=23)$ had no previous golf experience (Mean age $=$ 19.5 years, $S E=.21$ years). Skilled golfers $(n=19)$ had $2+$ years of high-school varsity golf experience or a PGA handicap of $\leq 8$ (Mean age $=19.4$ years, $S E=.43$ years). Skilled golfers were recruited using the same skill level criteria as previous work exploring the impact of speed instructions on execution (as a reference point, Beilock \& Gonso, 2008, reported a mean PGA handicap of about 6). The constraints of our putting green only allowed us to capture participants' entire body and club swing via a video camera if they were performing the putting task from a right-handed position. Thus, only right-handed individuals served as study participants. All participants were undergraduate students at a Midwestern University.

\section{Procedure and Design}

After giving informed consent and reporting previous golf experience, participants were introduced to the putting task. The putting task was performed on a carpeted indoor green $(3 \mathrm{~m} \times$ $3.7 \mathrm{~m}$ ) using a standard golf putter and ball. Individuals were instructed to putt a golf ball as accurately as possible to a target, marked by a square of red tape, on which the ball was supposed to stop. Individuals putted from four different starting locations, at various angles and distances (two starting locations at $122 \mathrm{~cm}$, one at $137 \mathrm{~cm}$, and one at $152 \mathrm{~cm}$ ) from the target. All participants followed the same random alternation of putting from the four locations.

A light next to each putt's starting location was illuminated by the experimenter prior to that putt to indicate the next putt's starting location. The experimenter then rolled a ball to the participant to be used on that putt and the participant was instructed to place the ball on the starting location with one of their feet. Participants were informed that the specific putting instructions they received (see speed and accuracy instructions below) should be followed once the ball was stationary on the starting position. This instruction provided a clear time frame for the start of each trial for the participant and a clear time frame for the recording of putting time from the videotapes of performance.

After each putt, the experimenter measured the distance $(\mathrm{cm})$ from the center of the target that the ball had stopped and then rolled a new ball to the participant. Individuals took 20 practice putts to familiarize themselves with the task. Individuals then took five blocks of 20 putts under accuracy instructions and five blocks of 20 putts under speed instructions for a total of 200 putts. The order of these 10 putting blocks was counterbalanced across participants. Specifically, four block orders were created with the constraint that participants had to take part in two speed and two accuracy blocks in the first half of the experiment (i.e., within the first five putting blocks) and two speed and two accuracy blocks in the last half of the experiment (i.e., within the last five blocks). Individuals were randomly assigned to one of the four block orders. Prior to the first putt of each block, and every fifth putt within a block, participants were reminded of the instructions for that particular block (see below). Between each block, individuals took a short break in which they were instructed to remain standing.

Accuracy instructions. Participants were told: "In this set of putts I want you to try to make the ball stop on the target. You can take as much time as you need in order to do this."

Speed instructions. Participants were told: "In this set of putts I want you to try to make the ball stop on the target. I have one additional instruction. I want you to try and take your putt as quickly as possible, but still make sure you are being as accurate as you can. Timing will begin when the ball is stationary on the starting position. Just try and take the putt as quickly as possiblefrom when the ball is stationary on the marker-while continuing to be as accurate as possible."

All putts were videotaped to capture putting time. The video camera was set up to the right and perpendicular to participants, approximately $2.5 \mathrm{~m}$ away so that the participant's head, the entire putter, and the four starting positions were in view. Prior to beginning the experiment, individuals were informed that their putting performance would be videotaped. Participants were further informed that this tape was for experimental records only and 
would not be shown to anyone who was not directly involved in the research. The video camera remained on throughout the entire experiment. Following the putting task, participants were reimbursed for their time, thanked, and debriefed.

\section{Results}

\section{Putting Performance}

Putting performance was measured by the distance (in $\mathrm{cm}$ ) away from the center of the target that the ball stopped after each putt (with smaller distances equaling better performance). Mean distance from the target was computed for the 100 putts in each instruction condition.

A 2 (expertise: novice, skilled) $\times 2$ (instruction: accuracy, speed) ANOVA on putting performance revealed a main effect of expertise, $F(1,40)=49.75, p<.01, M S E=33.86, \eta_{\mathrm{p}}^{2}=.55$, in which skilled golfers were better putters overall than novices. More importantly, there was also a significant expertise $\mathrm{x}$ instruction interaction, $F(1,40)=55.54, p<.01, M S E=7.83, \eta_{\mathrm{p}}^{2}=.58$. As can be seen in Figure 1, novices performed better under accuracy in comparison to speed instructions, $t(22)=5.31, p<$ $.01, d=.97$. Skilled golfers showed the opposite pattern, performing better under speed than accuracy instructions, $t(18)=6.40$, $p<.01, d=1.11$.

If an increase in execution time affords an increase in the deployment of attentional resources to the planning and unfolding of execution, novices should benefit from conditions that augment such attentional control (i.e., accuracy instructions) in comparison to conditions that limit it (i.e., speed instructions). Skilled golfers should show the opposite pattern. This is precisely what was found. We now turn to the time individuals spent putting to (a) ensure that our speed versus accuracy instructions were successful in altering performance time and (b) to examine where in the actual unfolding of performance, time constraints exerted their impact.

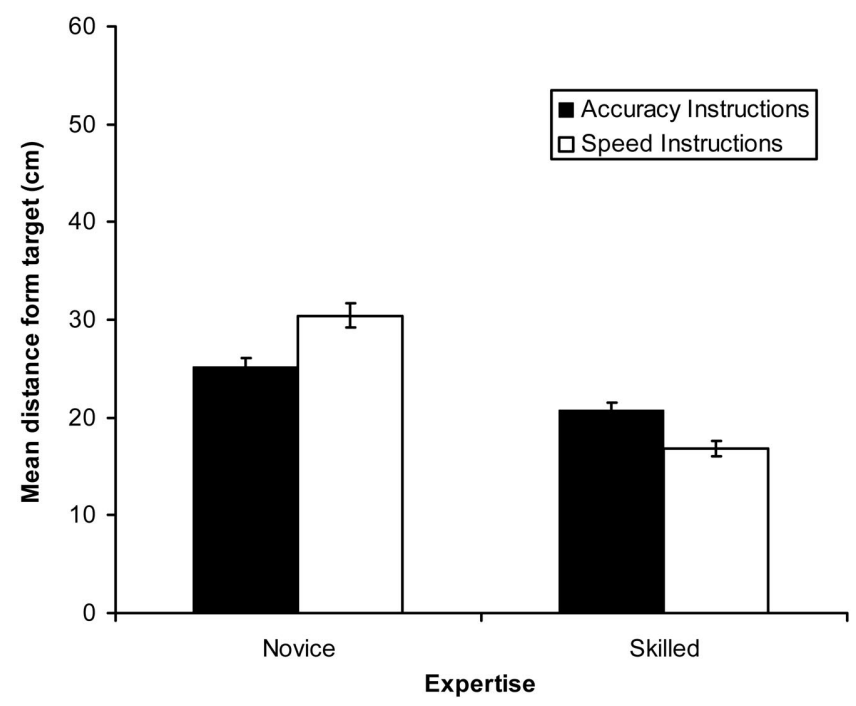

Figure 1. Mean putting performance (an error score) for the novice and skilled golfers under the speed and accuracy instructions in Experiment 1. Error bars are standard errors.

\section{Putting Time}

The videotapes of participants' putts were analyzed by one of four observers who were unaware of the experimental conditions or hypotheses. The videos were digitized, and each putt was coded on a frame-by-frame basis to yield a measurement of overall putting time (i.e., the time from when the ball was first stationary on the starting position until ball contact) and separate measures of preparation and movement time (to be explained below). Each coder was trained until their coding of at least two practice participants correlated with the times coded by the "trainer" at a level of .98 or higher.

Figure 2 reveals that our timing manipulation was successful. A 2 (expertise: novice, skilled) $\times 2$ (instruction: accuracy, speed) ANOVA on overall putting time revealed a main effect of instruction, $F(1,40)=178.65, p<.01, M S E=.63, \eta_{\mathrm{p}}^{2}=.82$. Individuals putted faster under speed than accuracy instructions. There was also a main effect of expertise, $F(1,40)=26.54, p<.01$, $M S E=1.88, \eta_{\mathrm{p}}^{2}=.40$, in which skilled golfers took more time to putt than novices. This is consistent with our previous golf work (Beilock, Bertenthal, McCoy, \& Carr, 2004) and with findings across a wide variety of skill domains demonstrating that experts often take longer to plan and execute their skill than novices (e.g., in physics problem solving and categorization tasks, see Chi et al., 1981). There was also a significant instruction $\times$ expertise interaction, $F(1,40)=6.56, p<.02, M S E=.63, \eta_{\mathrm{p}}^{2}=.14$, in which skilled golfers decreased their putting time by a greater amount than novices under the speed relative to the accuracy instructions.

As mentioned above, putts were further divided into preparation time and movement time components. Preparation time reflected the time from when the participant placed the ball on the starting position with his or her foot to the onset of club movement (i.e., the beginning of the backswing). Movement time reflected the time from the onset of club movement at the beginning of the backswing to ball contact. The follow through (which occurs after ball contact) was not included in the movement component of the putt. Although there are a number of possible ways to segment the components of a golf putt, as a first step toward examining the differential impact of speed instructions on novice and skilled performance time, we chose to segment putting into two discrete phases. Moreover, given previous work exploring the impact of time constraints in a decision task involving no movement component at all (Johnson \& Raab, 2003; Raab \& Johnson, 2007), it seemed sensible to divide the putting task into those components that did not involve movement (i.e., preparation) and those that did (i.e., movement component). Table 1 shows the means of the time taken in the preparation and movement components for novice and skilled golfers in the speed and accuracy conditions.

To examine the specific effects our instructions had on the preparation and movement components, we first computed the percent of time taken in the speed relative to the accuracy condition (i.e., speed time/accuracy time) for the preparation component (Novice: $M=46.9 \%, S E=2.3 \%$; Skilled: $M=48.4 \%, S E=$ $3.2 \%$ ) and movement component (Novice: $M=75.8 \%, S E=$ $2.5 \%$; Skilled: $M=76.5 \%, S E=3.1 \%)$. These ratios were subjected to a 2 (expertise: novice, skilled) $\times 2$ (putting component: preparation, movement) ANOVA which revealed only a main effect of component, $F(1,40)=351.6, p<.01, M S E=.01$, $\eta_{\mathrm{p}}^{2}=.90$. There was a greater time decrease from the accuracy to 


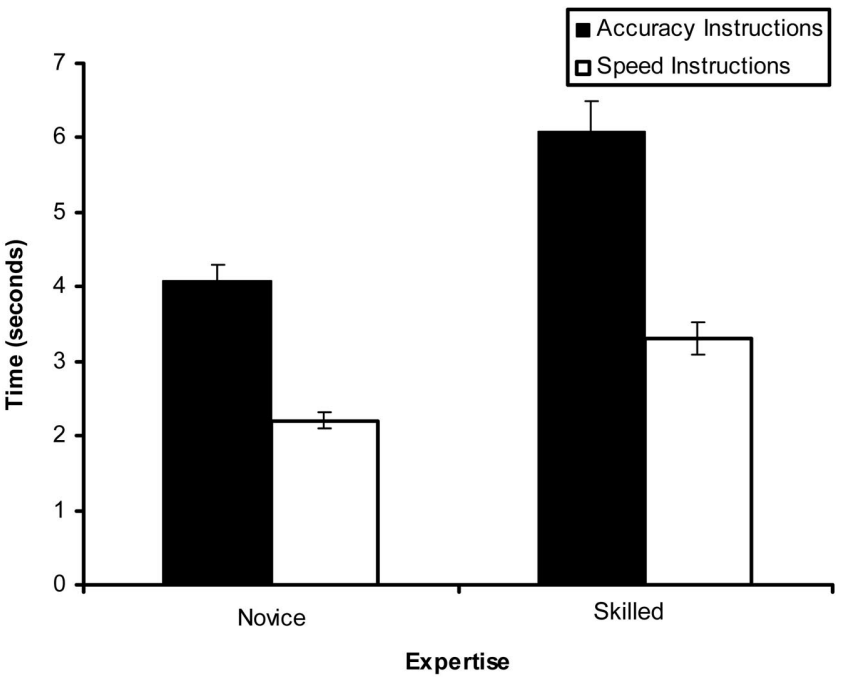

Figure 2. Mean putting time (seconds) for the novice and skilled golfers under the speed and accuracy instructions in Experiment 1. Error bars are standard errors.

speed instructions (indicated by a lower speed time/accuracy time percentage) in the preparation component of execution than in the movement component. This pattern of results suggests that the putting performance data reported above (in which putting outcomes differed as a function of speed vs. accuracy instructions for skilled and novice golfers) may be largely driven by changes in preparation time. To address this issue further, we next examined the direct relation between putting performance outcome and putting time as a function of golf putting skill level.

\section{Putting Time and Performance}

Overall putting time. We began by computing correlations (for each participant) between overall putting time (i.e., the time from when the ball was stationary on the starting position until ball contact) and putting performance (i.e., putting error; the distance that the ball landed from the center of the target) collapsed across all 200 trials - regardless of the putting instruction given. This type of correlation provides the most direct estimate of the relation between putting time and performance for each individual. These putting time/putting error correlations were next compared as a function of skill level, revealing a significant main effect of expertise, $F(1,40)=68.40, p<.01, M S E=.01, \eta_{\mathrm{p}}^{2}=.63$.

For novices, the average of the individual correlations between putting time and putting performance (computed as an error score) was negative, $r=-.15$, and significantly different from $0, t(22)=$ $6.56, p<.01$. The longer novices spent putting, the better they performed. The average of skilled golfers' individual correlations between putting time and performance was in the opposite direction, $r=.13$, and also significantly different from $0, t(18)=5.21$, $p<.01$. The longer skilled golfers spent putting, the worse they performed. Thus, the time spent putting impacted putting error differently as a function of skill level. Such a finding sets the stage to examine whether this putting time/putting error relation is driven more so by the time individuals spent in the preparation or the movement component of the putt or both.
Preparation versus movement time and performance. Correlations were computed separately for each participant for preparation time and putting error and movement time and putting error. We then submitted these individual putting time/putting error correlations to a 2 (expertise: novice, skilled) $\times 2$ (putting component: preparation, movement) ANOVA. This analysis produced a main effect of putting component, $F(1,40)=16.99, p<.01$, $M S E=.01, \eta_{\mathrm{p}}^{2}=.30$, and expertise, $F(1,40)=40.91, p<.01$, $M S E=.02, \eta_{\mathrm{p}}^{2}=.51$, which were qualified by a significant expertise $\times$ component interaction, $F(1,40)=15.39, p<.01$, $M S E=.01, \eta_{\mathrm{p}}^{2}=.29$.

For novices, the average of the individual correlations between preparation time and putting error and movement time and putting error were both negative, $r$ 's $=-.14$, and significantly different from $0, t(22)=6.40, p<.01$ and $t(22)=5.13, p<.01$, respectively. The longer novices spent either preparing or actually executing their putting stroke, the better they performed. For skilled golfers, the average of the individual correlations between preparation time and performance was in the opposite direction, $r=.15$, and also significantly different from $0, t(18)=6.04, p<$ .01. The longer skilled golfers spent preparing, the worse they performed. However, the correlation between skilled golfers' movement time and putting performance was $r=0$. This lack of correlation between movement time and putting error was not due to low variability in movement time for the skilled golfers. In fact, if anything, the novices showed slightly less variability in their movement time than skilled golfers (see Table 1).

\section{Discussion}

Experiment 1 replicates Beilock, Bertenthal, McCoy, \& Carr's (2004) findings demonstrating a differential impact of speed instructions on execution as a function of skill level. This can be seen in (a) the differences in putting performance outcomes under the speed versus accuracy instruction conditions for novice and skilled golfers and (b) the opposite correlations between putting time and performance as a function of golf expertise. In addition, Experiment 1 elucidates where in the putting process speed instructions exert their impact and how the relation between different putting components and putting performance outcomes change as a function of skill level. For novices, the longer time spent putting, the better the performance outcome. This relation held for both the preparation and movement time components of execution. For skilled golfers, the opposite pattern was observed. However, this was only true for the preparation component of execution.

Table 1

Preparation and Movement Time for Novice and Skilled Golfers in the Speed and Accuracy Conditions in Experiment 1

\begin{tabular}{|c|c|c|}
\hline & \multicolumn{2}{|c|}{ Time } \\
\hline & Preparation (s) & Movement (s) \\
\hline \multicolumn{3}{|l|}{ Accuracy } \\
\hline Novice & $M=2.94, S E=.17$ & $M=1.15, S E=.05$ \\
\hline Skilled & $M=4.46, S E=.36$ & $M=1.62, S E=.08$ \\
\hline \multicolumn{3}{|l|}{ Speed } \\
\hline Novice & $M=1.35, S E=.09$ & $M=0.86, S E=.04$ \\
\hline Skilled & $M=2.07, S E=.16$ & $M=1.24, S E=.07$ \\
\hline
\end{tabular}


Why were the putting outcomes of novices associated with both preparation and movement time, while skilled golfers' outcomes were only associated with preparation time? To the extent that part of what becoming an expert entails is the development of proceduralized routines that run largely outside of explicit attentional control (Anderson, 1983, 1993; Fitts \& Posner, 1967), then the time in which performance is open to influence may be during the initial planning and fine-tuning of the action rather than during the actual unfolding of execution. Once initiated, skilled performers' actions may be encapsulated in such a way that any attempt to control performance by changing execution time may not have much of an effect on the performance outcome.

Such an explanation is consistent with work by Gray (2004) who demonstrated that expert baseball players were much worse than their novice counterparts in identifying (during the real-time unfolding of execution) the direction that their swing was traveling when prompted by an auditory probe. If the real-time unfolding of performance is not open to introspection of movement direction for Gray's baseball players, then it may not be open to instructional or attentional modulation for our golfers as well.

Added support for the above notion comes from a distinction that has been made in the motor learning and control literature between the planning of actions and their online control (Glover \& Dixon, 2002). According to this planning-control distinction, the planning of an action is susceptible to interference from cognitive and perceptual variables that the online control of an action is not. This is because once an action is instantiated, it is believed to be yoked to the visual-spatial properties of its target which allows for automatic corrections for the influence of any interfering variables. This planning-control distinction has found support in a number of well-learned skills such as reaching and grasping toward targets (see, e.g., Glover, Rosenbaum, Graham, \& Dixon, 2004). In terms of the current work, it may be that for a well-learned golf putt, the close link between the movement component of execution and the putting target largely eliminates the influence of speed instructions on the relation between movement execution and actual putting outcomes.

Moreover, in discrete skills ranging from archery to rifle shooting to golf, the neural processes involved in the preparation component of execution have often been shown to be the best predictors of actual performance outcomes-even more than those processes underlying the movement component of execution itself (Deeny et al., 2003; Hatfield \& Hillman, 2001; Janelle et al., 2000; Vickers, 1996). Such findings have been taken to suggest that the quality of sensorimotor performance of highly skilled individuals "lies at the level of the organization of neural networks during motor planning" (p. 804, Milton, Solodkin, Hlustik, \& Small, 2007). Given the above evidence, it is perhaps not surprising that, in the current work, the strongest predictor of actual putting outcomes in our skilled golfers is putting preparation time.

A prominent notion derived from the skill acquisition and automaticity literature is that individuals relinquish explicit attentional control of execution processes as practice accumulates and skill learning progresses (e.g., Anderson, 1982; Fitts \& Posner, 1967). However, such movement may not be so unidirectional in that skilled performers may "continuously cycle" through different modes of attentional control (Gray, 2004). Although these cycles may be exaggerated under severe or stressful conditions (e.g., a high pressure situation that prompts explicit attention to execu- tion), they are also thought to naturally occur during the course of normal performance in nonstress situations. For example, Gray (2004) found that skilled baseball players performing a hitting task in a pitching simulator went through periods of more or less explicit control of their hitting skill which was correlated with less accurate and more accurate hitting performance, respectively. The current work points to one variable which may contribute to naturally occurring skilled performance variability-the time taken in the preparation component of skill execution. Less preparation time may lead to a higher likelihood of activating and executing the type of well-practiced motor program that governs performance when it is at its most optimal level. With unlimited performance time, skilled performers may recompute and compile new skill execution processes or reparameterize or retune existing ones. This may create opportunities for error that were not present in the previously compiled control structure.

Of course, more work is needed to explicate the precise mechanisms by which unlimited performance time negatively impacts the high-level performance of skilled golfers. Future research in which, for example, golfers are probed about specific aspects of online performance during speed and accuracy instructions may help to do just this. For example, in Gray's (2004) work mentioned above, it was found that highly skilled baseball players batted at a lower level under high-pressure as compared to low-pressure conditions. However, these players were actually better able to introspect on component processes of their performance (e.g., make judgments about whether their bat was traveling upward or downward at a particular moment in time) under the stressful as compared to the nonstressful situation. The high-pressure situation likely prompted attention to execution which, although disrupting well-learned performance processes, afforded access to components of performance that were otherwise largely closed to explicit introspection and report (Beilock \& Carr, 2001). Similar to Gray's batters, it may be that when given unlimited performance time, skilled golfers will be better able to make judgments about specific aspects of their performance yet will perform less accurately than in situations in which time constraints are imposed. Future work designed to test this notion will shed additional light on the psychological mechanisms by which time constraints (or a lack thereof) exert their impact.

\section{Experiment 2}

If the impact of time constraints on putting performance (and specifically the reverse speed-accuracy trade-off seen for skilled and not novice individuals) is truly driven by performance time's modulation of attentional control, then conditions that prompt skilled golfers to allocate attention to performance (and, in light of Experiment 1's results, specifically to the planning, preparation, and initial compilation of performance) should alter the impact that speed instructions have on putting outcomes. Such a finding would not only demonstrate the susceptibility of skilled performance to environmental changes, but might also highlight the ability of speed instructions to diagnose execution differences as a function of skill level. Experiment 2 explored these ideas.

From a skill acquisition and automaticity perspective, novice golfers must devote explicit attentional resources to performance. Thus, the more time available to deploy such resources, the better. In contrast, the proceduralized processes of experts are thought to 
be well established such that the selection of optimal action processes occurs relatively immediately (Klein et al., 2005) and less performance time limits the counterproductive tendency to monitor and try to control or adjust these processes (Beilock, Bertenthal, McCoy, \& Carr, 2004). As a result, limiting performance time benefits skilled performers-a reverse speed-accuracy trade-off.

But what happens when experts are asked to perform their skill in an unfamiliar environment or while wielding an unfamiliar tool? To the extent that a new tool or novel performance environment prompts experts to try to control their behavior in much the same fashion as a novice (e.g., by constructing a new procedure from scratch whose parameters are open to explicit attentional scrutiny during preparation, execution, or both), then we may see a very different impact of speed instructions to that reported above. Of course, whether skilled golfers wielding new tools are initially negatively impacted by speed instructions to the same extent as novices, and if so, how skilled performers adapt to such constraints, is unknown. An understanding of the relative time frame by which skilled performers adapt to the constraints of novel tools is important as it will provide insight into the precise ramifications of changing the tools of one's trade.

In Experiment 2 we had skilled and novice golfers perform the same putting task as in the previous experiment but rather than using a normal putter, we asked our participants to use a funny putter made from an s-shaped and arbitrarily weighted putter shaft. If speed instructions exert their impact by altering one's ability to deploy attention to preparation and/or movement, then skilled golfers operating with the funny putter should be negatively impacted by time constraints - at least until they have adapted to the novel constraints of the putter. Because novices are not accustomed to putting with any type of putter, the impact of speed instructions on novice performance should look much like that seen in Experiment 1.

\section{Method}

\section{Participants}

Novices $(n=20)$ had no previous golf experience $(M$ age $=$ 19.6 years, $S E=.36$ years $)$. Skilled golfers $(n=20)$ had $2+$ years of high-school varsity golf experience or a PGA handicap of $\leq 8$ ( $M$ age $=20.4$ years, $S E=.34$ years $)$. As in Experiment 1, everyone putted right-handed, and all participants were undergraduate students at the same Midwestern University as that used in the first experiment. None of the participants in Experiment 2 took part in Experiment 1. Moreover, in an attempt to ensure similar skill levels across experiments, the novice and skilled golfers in Experiment 2 were drawn from the same participant population, using the exact same inclusion criteria as Experiment 1.

\section{Procedure and Design}

The procedure was the same as Experiment 1 with the exception that all participants completed the experiment with the funny putter rather than with a regular putter.

\section{Results}

\section{Putting Performance}

As in Experiment 1, putting performance was measured by the distance $(\mathrm{cm})$ away from the center of the target that the ball stopped after each putt. We began by computing mean accuracy for the 100 putts in the speed instruction condition and the 100 putts in the accuracy instruction condition.

A 2 (expertise: novice, skilled) $\times 2$ (instruction: accuracy, speed) ANOVA revealed main effects of expertise, $F(1,38)=$ $31.33, p<.05, M S E=160.69, \eta_{\mathrm{p}}^{2}=.45$, and instruction, $F(1$, $38)=14.17, p<.05, M S E=44.67, \eta_{\mathrm{p}}^{2}=.27$, which were qualified by an expertise $\times$ instruction interaction, $F(1,38)=$ 17.89, $p<.05, M S E=44.67, \eta_{\mathrm{p}}^{2}=.32$. As seen in Figure 3, novices performed better under accuracy in comparison to speed instructions, $t(19)=4.14, p<.01, d=.66-$ the same pattern seen in Experiment 1. Skilled golfers showed no difference as a function of instruction, $t(19)=0.89$. Thus, novice golfers exhibited worse putting performance under conditions in which time constraints were imposed (i.e., speed instructions) in comparison to conditions in which such constraints were not an issue (i.e., accuracy instructions). This result replicates Experiment 1 and is not surprising given that novices should perform in a similar manner regardless of putter. To a novice, all putters are novel. In contrast skilled individuals' putting performance was not differentially impacted by our timing instructions, at least when performance was averaged across all trials in each instruction condition.

Is it possible that skilled golfers are able to adapt to the novel demands of the funny putter rather rapidly with practice? To the extent that skilled performers are able to utilize some of their golf putting expertise in learning to wield this new tool, then these individuals may adapt to and successfully utilize the novel constraints of the funny putter over the course of our 200 trial experiment. If so, then the speed and accuracy instructions might

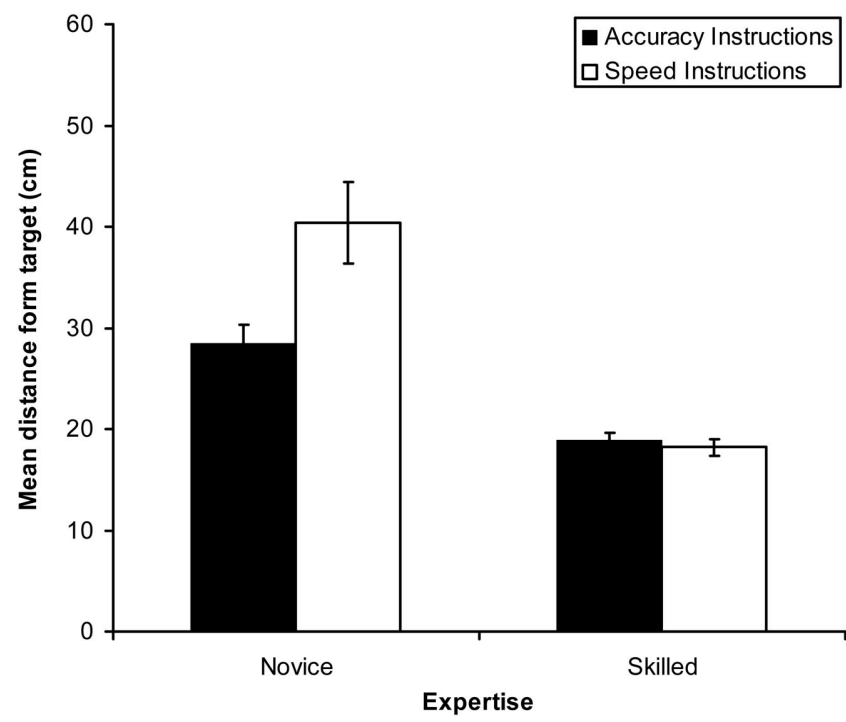

Figure 3. Mean putting performance (an error score) for the novice and skilled golfers under the speed and accuracy instructions in Experiment 2. Error bars are standard errors. 
have quite different effects on performance as a function of whether one is looking at the early trials in each instruction condition or at the later trials.

To address this issue, we compared performance in the first two accuracy and first two speed blocks (early trials) with performance in the last two accuracy and last two speed blocks (late trials). The middle block of putts in each instruction condition was omitted to equate the number of putts in the early and late trials. Note, however, that leaving this middle block in would not have changed the pattern of results in any way. As seen in Figure 4, a 2 (expertise: novice, skilled) $\times 2$ (instruction: accuracy, speed) $\times$ 2 (practice: early trials, late trials) ANOVA revealed a significant three-way interaction, $F(1,38)=6.58, p<.05, M S E=$ $13.12, \eta_{\mathrm{p}}^{2}=.15$.

For novices, a 2 (instruction: accuracy, speed) $\times 2$ (practice: early trials, late trials) ANOVA on putting performance (i.e., an error score) revealed a main effect of practice, $F(1,19)=8.75$, $p<.01, M S E=19.25, \eta_{\mathrm{p}}^{2}=.32$, and a main effect of instruction, $F(1,19)=18.23, p<.05, M S E=19.25, \eta_{\mathrm{p}}^{2}=.49$, but no practice $\times$ instruction interaction, $F<1$. Regardless of practice, novices putted better under accuracy in comparison to speed instructions. From our perspective, this suggests that the amount of practice in the current experiment was not enough to change how novices allocated attention to execution. As a result, the impact of the speed and accuracy conditions on performance did not vary with time. This is not surprising given the tens of thousands of hours of practice it is thought to take to become an expert performer (Ericsson \& Charness, 1994).

For skilled golfers, the same ANOVA revealed a significant practice $\times$ instruction interaction, $F(1,19)=35.68, p<.05$, $M S E=6.99, \eta_{\mathrm{p}}^{2}=.65$. As seen in Figure 4, in the early trials,

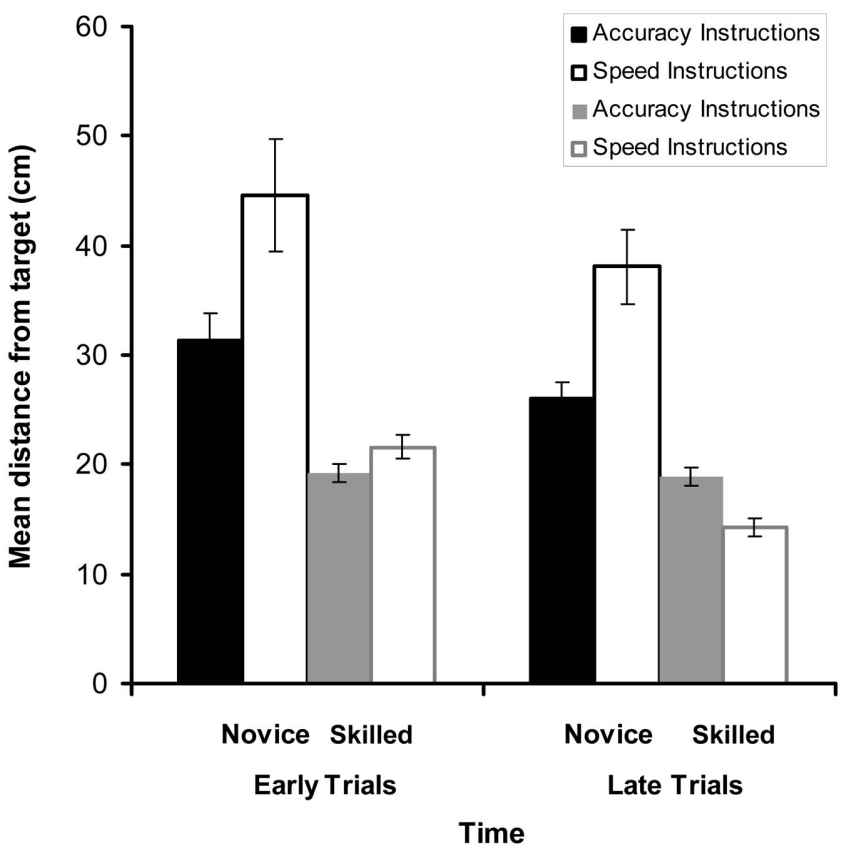

Figure 4. Mean putting performance (an error score) for the novice and skilled golfers under the speed and accuracy instructions for both the early and late putting blocks in Experiment 2. Error bars are standard errors. skilled golfers putted better under accuracy than speed instructions, $t(19)=2.37, p<.05, d=.55$. In the later trials, skilled golfers showed the opposite pattern, $t(19)=4.72, p<.05, d=$ 1.18. Thus, skilled golfers' high-level skill allowed them to readily adapt to the constraints of our funny putter. Early in practice, skilled golfers were harmed by speed conditions that presumably limited the deployment of attention to the unfamiliar putting task. Later in practice, these same time constraints actually benefited performance. It should be noted that after demonstrating practice differences in Experiment 2, we went back and added this practice variable to all the analyses in Experiment 1. There were no interactions with practice in Experiment 1 . We next turn to an examination of putting time to (a) ensure that our instructions produced their desired effect in terms of changing the time individuals spent putting and (b) examine the direct relation between putting time and putting outcome as a function of golf putting expertise and task practice.

\section{Putting Time}

A 2 (expertise: novice, skilled) $\times 2$ (instruction: accuracy, speed) $\times 2$ (practice: early trials, late trials) ANOVA on overall putting time (i.e., the time from when the ball was placed on the starting position until ball contact) revealed a main effect of instruction, $F(1,37)=196.85, p<.01, M S E=.77, \eta_{\mathrm{p}}^{2}=.84$, in which all participants performed faster under speed than accuracy instructions, a main effect of expertise, $F(1,37)=15.14, p<.01$, $M S E=5.29, \eta_{\mathrm{p}}^{2}=.29$, in which skilled golfers took longer to putt than novices, and a main effect of practice, $F(1,37)=$ $13.49, p<.01, M S E=.62, \eta_{\mathrm{p}}^{2}=.27$. Putting time was longer in the early versus later trials. There was also a significant instruction $\mathrm{x}$ practice interaction, $F(1,37)=13.31, p<.01$, $M S E=.20, \eta_{\mathrm{p}}^{2}=.27$, in which the timing instructions had more of an impact on putting time in the early versus late trials (see Figure 5 and note that the degrees of freedom are reduced here because partial timing data were lost for one novice). No other interactions reached significance.

We next examined the specific effects of our timing instructions on the preparation and movement components of execution as a function of practice and expertise. See Table 2 for the means of the time taken in the preparation and movement components for novice and skilled golfers in the speed and accuracy conditions in the early and late putting blocks.

We began by computing the percent of time allocated to the speed relative to the accuracy condition (i.e., speed time/accuracy time) in the early putting blocks for the preparation component (Novice: $M=42.4 \%, S E=4.1 \%$; Skilled: $M=53.0 \%, S E=$ $3.7 \%$ ) and movement component (Novice: $M=75.2 \%, S E=$ $2.8 \%$; Skilled: $M=83.9 \%, S E=2.2 \%)$ and the later putting blocks for the preparation component (Novice: $M=47.2 \%, S E=$ 5.7\%; Skilled: $M=58.0 \%, S E=4.6 \%$ ) and movement component (Novice: $M=76.2 \%, S E=3.2 \%$; Skilled: $M=86.4 \%, S E=$ $2.1 \%)$. These percentages were subjected to an expertise x putting component $\times$ practice ANOVA.

This analysis revealed a main effect of practice, $F(1,37)=4.74$, $p<.04, M S E=.01, \eta_{\mathrm{p}}^{2}=.11$. There was a greater decrease in putting time from the speed to accuracy instructions (indicated by a lower speed time/accuracy time percentage) in the early relative to the late putting blocks. There was also a main effect of exper- 


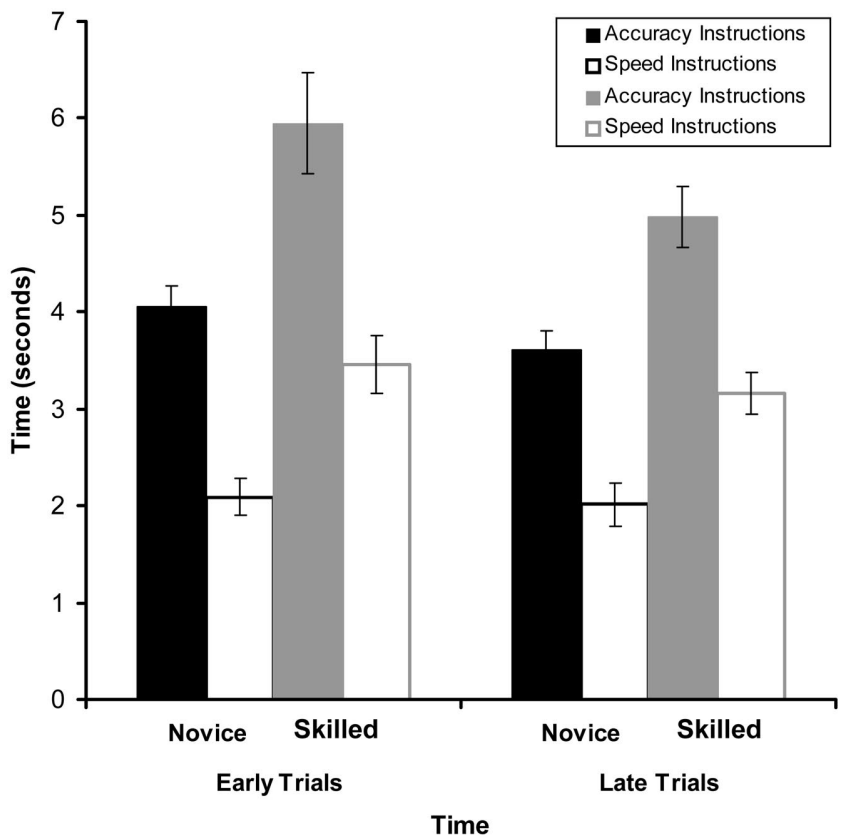

Figure 5. Mean putting time (seconds) for the novice and skilled golfers under the speed and accuracy instructions for both the early and late putting blocks in Experiment 2. Error bars are standard errors.

tise, $F(1,37)=5.05, p<.04, M S E=.08, \eta_{\mathrm{p}}^{2}=.12$. Novices decreased their putting time by a greater percent than skilled individuals. Finally, there was a main effect of putting component, $F(1,37)=188.17, p<.01, M S E=.02, \eta_{\mathrm{p}}^{2}=.84$, in which there was a greater percentage time decrease from the accuracy to speed instructions in the preparation component of execution than in the movement component. No interactions reached significance. To explore the direct relation between putting time and performance outcome as a function of skill level, practice, and putt component, we next looked at the correlations between the time spent putting and putting outcomes.

\section{Putting Time and Performance}

Overall putting time. As in Experiment 1, we began by computing (for each participant) correlations between overall putting time (i.e., the time from when the ball was stationary on the starting location to ball contact) and putting performance outcome (i.e., an error score). To examine how these correlations differed as a function of practice, we computed separate correlations for the early versus the later putting trials. These correlations were submitted to a 2 (expertise: novice, skilled) $\times 2$ (practice: early trails, later trials) ANOVA. This analysis revealed a main effect of expertise, $F(1,38)=23.89, p<.01, M S E=.06, \eta_{\mathrm{p}}^{2}=.39$, and a main effect of practice, $F(1,38)=8.81, p<.01, M S E=.02, \eta_{\mathrm{p}}^{2}=.19$, which were qualified by a significant practice $\mathrm{x}$ expertise interaction, $F(1,38)=15.54, p<.01, M S E=.02, \eta_{\mathrm{p}}^{2}=.29$.

For novices, the average of the individual correlations between putting time and putting performance outcome in both the early $(r=-.21)$ and later trials $(r=-.24)$ did not significantly differ from each other, $F<1$, but were significantly different from 0 , $t(19)=4.35, p<.01$, and $t(19)=5.39, p<.01$, respectively. The more time novices spent putting, the better they performed. By contrast, the average correlations for skilled golfers in the early $(\mathrm{r}=-.05)$ and later trials $(r=.14)$ were significantly different from each other, $F(19)=19.11, p<.01, M S E=.02$. In the earlier trials, the slight negative correlation between putting time and performance (i.e., the longer the putting time, the better the outcome) was not significantly different from $0, t(19)=1.33, p=$ .20 , suggesting no significant relation between putting time and performance outcome. However, once skilled golfers had adapted to the novel constraints of the funny putter in the later trials, increased putting time was related to worse performance outcomes. This correlation was significantly different from $0, t(19)=$ $3.37, p<.01$.

Preparation time versus movement time and performance. We next computed the same putting time/putting error correlations reported above, but separately for both the time involved in the preparation and movement components. Moreover, given the differences as a function of task practice reported above, we computed the putting time/putting error correlations separately for the early and late trials.

Early in practice, a 2 (expertise: novice, skilled) $\times 2$ (putting component: preparation, movement) ANOVA on the putting time/ putting error correlations revealed only a main effect of expertise, $F(1,38)=6.62, p<.02, M S E=.06, \eta_{\mathrm{p}}^{2}=.15$. As seen in the overall putting time analyses above, novices showed a stronger negative correlation than skilled golfers between overall putting time and performance outcome (i.e., the longer time spent putting, the better participants performed) — as a reminder, putting performance outcome was computed as an error score.

By contrast, the same ANOVA later in practice revealed a main effect of expertise $F(1,38)=32.04, p<.01, M S E=.06, \eta_{\mathrm{p}}^{2}=$ .46 , which was qualified by a significant putting component $\mathrm{x}$ expertise interaction, $F(1,38)=4.73, p<.04, M S E=.01, \eta_{\mathrm{p}}^{2}=$ .11. Novices showed negative correlations between both preparation time and putting error $(r=-.22)$ and movement time and putting error $(r=-.19)$. Both of these correlations were significantly different from $0, t(19)=5.1, p<.01$, and $t(19)=4.0, p<$

Table 2

Preparation and Movement Time for the Novice and Skilled Golfers in the Speed and Accuracy Conditions in the Early and Late Putting Blocks in Experiment 2

\begin{tabular}{lll}
\hline & \multicolumn{2}{c}{ Time } \\
\cline { 2 - 3 } & \multicolumn{1}{c}{ Preparation (s) } & Movement (s) \\
\hline Accuracy & & \\
$\quad$ Early putting blocks & & \\
$\quad$ Novice & $M=2.98, S E=.22$ & $M=1.07, S E=.04$ \\
$\quad$ Skilled & $M=4.56, S E=.47$ & $M=1.39, S E=.08$ \\
$\quad$ Late putting blocks & $M=2.55, S E=.22$ & $M=1.05, S E=.03$ \\
$\quad$ Novice & $M=3.60, S E=.27$ & $M=1.39, S E=.07$ \\
$\quad$ Skilled & & \\
Speed & $M=1.29, S E=.16$ & $M=0.80, S E=.04$ \\
Early putting blocks & $\quad M=2.32, S E=.26$ & $M=1.15, S E=.06$ \\
$\quad$ Novice & & \\
$\quad$ Skilled & & \\
Late putting blocks & $M=1.22, S E=.20$ & $M=0.80, S E=.04$ \\
$\quad$ Novice & $M=1.98, S E=.19$ & $M=1.18, S E=.05$ \\
$\quad$ Skilled & & \\
\hline & &
\end{tabular}


.01 , respectively. Skilled golfers, on the other hand, showed a very different pattern of results. Namely, once skilled golfers had practiced with the new putting tool, they showed a positive relation between putting time and error for the preparation component of execution, $r=.15$, which was significantly different from $0, t(19)=$ $3.29, p<.01$, and no significant relation between movement time and putting error, $r=.05, t(19)=1.59$, ns. Thus, similar to Experiment 1, once skilled golfers were fairly well practiced with the funny putter, the less time spent preparing to putt, the better the performance outcome.

\section{Discussion}

When skilled golfers are given a novel putter that forces them to attend to their performance, they suffer under time constraints-at least until they have adapted to the new club requirements. In the early trials, speed instructions were associated with worse performance for skilled golfers. However, in the later trials, the opposite effect occurred. Previous work has demonstrated that the funny putter manipulation prompts experts to allocate attention to putting in much the same way as a novice (Beilock, Wierenga, \& Carr, 2002). If the impact of time constraints on putting performance is largely driven by the modulation of attentional control, then conditions that prompt experts to allocate attention like novices should alter the impact that our speed instructions have on skilled performance-at least until these skilled individuals have adapted to the constraints of the funny putter. This is the result we observed.

The details of these trends point to some more specific conclusions about the mechanisms that change with practice as expertise develops. Early in practice with the funny putter, skilled golfers' putting performance was harmed by speed instructions. The reverse pattern was seen in the later trials after the skilled golfers had practice with our novel tool-but only for the preparation component of execution. For skilled golfers performing in practiced environments, the time in which putting outcomes appear to be open to influence is during this initial compilation of the action (sparked by an intention to act) rather than during the actual unfolding itself. This is consistent with the findings of Experiment 1 and previous work demonstrating that (a) skilled performers have difficulty introspecting on the actual unfolding of execution (Gray, 2004), (b) cognitive and perceptual variables impact the planning more so than the online execution of familiar tasks (Glover, Rosenbaum, Graham, \& Dixon, 2004), and (c) in discrete skills ranging from rifle shooting to golf, the neural processes involved in the preparation component of execution correlate strongly with actual performance outcomes (Hatfield \& Hillman, 2001; Janelle et al., 200; Vickers, 1996). It may be that unlimited performance time prompts skilled golfers to compute new performance processes and procedures, or reparameterize existing procedures unnecessarily, rather than retrieving a wellpracticed motor program that governs performance when individuals are performing at an optimal level. Such a recomputation likely creates opportunities for error that were not present in the previously compiled and well-practiced control structure.

\section{General Discussion}

In two experiments we show that the skill level of the performer, either manipulated via task experience (as in Experiment 1) or by novel task demands (as in Experiment 2) carries implications for the impact of speed instructions on performance. Novice performance is thought to demand the explicit allocation of attention to the selection and implementation of skill processes and procedures (Anderson, 1993; Beilock \& Carr, 2001; Fitts \& Posner, 1967). As a result, conditions that limit the deployment of attention harm this skill group relative to conditions that do not impose such constraints. By contrast, expert performance is believed to operate largely outside of conscious awareness (Beilock \& Carr, 2001; Gray, 2004). If putting faster affords less time for the planning, selection, fine-tuning, and control of execution, then at least under well-practiced conditions, time constraints may benefit skilled individuals by preventing the explicit allocation of attention to skill processes and procedures that are best left unattended.

When, however, the constraints of performance are altered such that skilled individuals find themselves operating in environments consisting of novel task components (e.g., the funny putter), the impact of speed instructions on performance change. Although novice funny putter performance did not deviate from regular putter performance (novices are not accustomed to putting with any type of putter), skilled golfers operating with the funny putter were negatively impacted by time constraints. This negative impact, however, was limited to early in practice when skilled individuals had not adapted to the novel constraints of the putter. An understanding of the relative time frame by which skilled performers adapt to novel task demands is important because it provides insight into the precise ramifications of changing the tools of one's trade. And indeed, when such tools do change in real world venues, there are often unanticipated consequences. Take, for example, the National Basketball Association's (NBA) 2006 change to a new basketball consisting of a new design and material (National Basketball Association, 2006). Immediately, the athletes complained about adapting to the new constraints of their tool and the ball was promptly switched back. What is unknown, however, is whether with practice, the new ball would be satisfactory to the players. The relatively short amount of practice it took our skilled golfers to adapt to the funny putter, and the reversal of the impact of speed instructions on performance outcomes as a function of putting block, suggest that this may be the case. Of course, future research is needed to look at the precise boundary conditions by which such changes take place. Nonetheless, this work suggests that successfully changing the tools of one's trade may be possible with a little practice.

The impact of speeded execution was not only evident from overall putting time-performance outcome relations, but also from the correlations between the durations of specific putting components and performance outcomes: Both the time spent in preparation and the time spent in movement were negatively correlated with putting error (i.e., the mean distance the ball landed from the target location) for unskilled individuals (i.e., novices in Experiment 1) or individuals operating with unfamiliar tools (i.e., novices, and skilled golfers using the funny putter in the early trials of Experiment 2). By contrast, individuals performing a well-learned putting task showed a positive correlation between putting time and putting error, but only for the preparation component of the putt (i.e., skilled golfers in Experiment 1 and in the late trials of Experiment 2). Thus, by not only looking at the relation between overall performance time and its outcome, but by looking within a 
single performance-at the relations between the timing of various components of execution and the outcome-we are able to demonstrate where exactly in the unfolding of performance speed instructions exert their impact.

One might notice that, overall, putting accuracy of the skilled golfers using the funny putter in Experiment 2 was not noticeably different than the putting accuracy of skilled golfers wielding the regular putter in Experiment 1. Given that the odd-shaped funny putter should harm the performance of skilled golfers relative to regular putter use (Beilock \& Carr, 2001), one might be concerned that the golfers in Experiment 2 were of a higher overall skill level than their Experiment 1 counterparts. However, the skilled performers in both experiments were drawn from the same participant population using the same inclusion criteria, thus this outcome does not seem likely. More importantly, it should be noted that - at least in the early trials - the speed and accuracy instructions were predicted to have opposite effects on skilled performers using the novel and familiar putting tools. And, this is exactly what occurred: Skilled golfers using the regular putter in Experiment 1 performed better under speed relative to accuracy instructions. Skilled golfers using the funny putter showed the opposite pattern. Thus, comparing performance in the early trials seems difficult given that the conditions under which individuals performed did not similarly impact performance. This was not the case in the latter putting blocks. Here, skilled golfers using either the regular or funny putter benefited from speed instructions. And, given that the skilled golfers in Experiment 2 had time to adapt to the novel constraints of the funny putter by these later trials, comparing performance later in practice as a means to assess similarity in skill level across experiments seems optimal. Specifically, to the extent that our skilled golfers were of similar expertise across experiments, there should not be a significant performance difference as a function of experiment in these later trials for either the accuracy instruction condition or the speed instruction condition, and this was true (accuracy instruction condition in Experiment 1: $M=$ $20.42 \mathrm{~cm}, S E=0.98 \mathrm{~cm}$; in Experiment 2: $M=18.90 \mathrm{~cm} ; S E=$ $0.91, F=1.32$, ns.; Speed instruction condition in Experiment 1: $M=15.73 \mathrm{~cm}, S E=0.71 \mathrm{~cm}$; in Experiment 2: $M=14.23, S E=$ $0.85), F=1.80, n s$.).

The current work uses the relation between instruction condition and performance outcomes-and more specifically, the relation between the timing of particular performance components (e.g., preparation) and putting outcomes-to infer differences in the cognitive control structures governing performance as learning progresses. Although our findings shed light on (a) differences in novice and skilled performance, (b) differences within a particular performance as it unfolds in real time, and (c) how putting time/ performance outcome relations change as a function of introducing a new tool of the trade, our work does leave open the possibility that attentional mechanisms other than the ones we propose are driving performance. For example, could the relation between longer performance time and putting accuracy for the skilled golfers wielding familiar tools be due to distraction from the task at hand rather than maladaptive attention to execution? Given that the same speed instructions had the opposite effect on novice golfers (i.e., novice golfers benefited from longer performance time), and novice golfers are known to be harmed by distraction, a distraction account does not seem probable. Nonetheless, future work employing independent measures of cognitive control over and above putting time-outcome relations will shed further light on the cognitive mechanisms governing performance and how such control structures change as learning progresses.

\section{Speeded Performance and its Implications}

The current work speaks to how speeded versus unspeeded execution relates to performance outcomes as a function of skill level and familiarity with one's tools of the trade. This work also suggests a means to diagnose the skill level of individuals performing in both the confines of the lab and the complexities of the real world. Specifically, coaches, teachers, and performers may gain insight into the attentional demands of skilled performance by examining how performers are impacted by the implementation of instructions that encourage faster performance. This may be useful for diagnosing changes across the learning continuum (whereby a decrease in the control of step-by-step performance is one hallmark of high-level execution; Anderson, 1983, 1993; Fitts \& Posner, 1967; Proctor \& Dutta, 1995). It may also play a role in assessing when one has reached a level of performance whereby explicit attentional control of step-by-step execution is no longer necessary to achieve desired performance outcomes following exposure to a new tool or new skill execution environments. A golfer, for example, interested in using a new club to achieve an optimal biomechanical swing, may need to expend additional executive resources concentrating and maintaining attentional focus on performance while learning the new swing parameters. The impact of speeded conditions on performance then may be one way in which to diagnose when the swing is corrected and the control structures of performance have returned to the proceduralized state that is one hallmark of well-practiced execution (Anderson, 1983, 1993; Beilock \& Carr, 2001; Fitts \& Posner, 1967). Although one might argue that one could merely ask performers whether they have achieved this fluent level of execution, given that some components of a skill may not be open to explicit introspection and report, and we are often unable to accurately assess our own learning (Kruger \& Dunning, 1999), a speed instruction manipulation may be a desired alternative for assessing skill level.

Not only may speeded performance conditions aid the diagnosticity of individuals' place along the learning continuum, increasing performance speed may be effective in combating the negative impact of high-stakes situations on well-learned or proceduralized performance processes. Explicit monitoring theories of choking under pressure suggest that suboptimal performance of a welllearned skill under pressure results from an attempt to exert explicit monitoring and control on proceduralized knowledge that is best initiated and run-off as an uninterrupted and unanalyzed structure (Baumeister, 1984; Beilock \& Carr, 2001; Beilock, Wierenga, \& Carr, 2002; Lewis \& Linder, 1997; Masters, 1992, 2000; Poolton, Masters, \& Maxwell, 2005). And, as mentioned above, previous work in our lab (Beilock, Bertenthal, McCoy, \& Carr, 2004; Beilock, Carr, MacMahon \& Starkes, 2002) and others (Castaneda \& Gray, 2007; Gray, 2004; Jackson, Ashford, \& Norsworthy, 2006) has found that conditions that prompt explicit attention to well-learned skills (e.g., golfers told to "monitor their swing and attempt to keep their club head straight as it travel(s) toward the target ..." Beilock, Bertenthal, McCoy, \& Carr, 2004) 
result in worse outcomes in comparison to conditions in which such skill-focus instructions are absent.

To the extent that high pressure situations operate by prompting attention to execution in a manner that brings processes once operating outside of working memory back into explicit attentional control, then situations that limit the ability to employ such resources may actually benefit performance. Given that attention takes time to deploy (Posner \& Snyder, 1975; Shiffrin \& Schneider, 1977), speed manipulations such as those used in the current work may actually work to inoculate individuals against the negative impact of pressure environments - at least in welllearned sensorimotor skills (see Beilock, Kulp, Holt, \& Carr, 2004, and Beilock \& Carr, 2005, for evidence that different kinds of skills differ in their response to pressure and other forms of stress).

One might assume that the impact of speed instructions would be reflected in the actual unfolding of skilled golfers' putting stroke (i.e., the movement component), rather than during the initial preparation and tuning of putting. However, skill-focused instructions such as those presented above direct attention to performance more generally (e.g., monitoring one's swing) in addition to directing attention to specific aspects of execution (e.g., keeping one's club head straight). Thus, it is unclear from previous work as to where in the actual unfolding of a skill these instructions exert their impact. The current work suggests that less preparation time benefits putting outcomes for skilled performers. With unlimited time, skilled performers may recompute and compile new skill execution processes or reparameterize or retune existing ones. This may create new opportunities for error that were not present in previously compiled procedures.

Of course, it is an open question as to how speed instructions would impact nonself paced tasks such as taking a jump shot in basketball or catching a baseball. On the one hand, given that actions other than the performer's determines preparation and execution time in such sports (e.g., an opponent making a move to steal the ball from a shooter about to go up for a jump shot in basketball may change the parameters for optimal shooting time), one might argue that speed instructions such as those imposed in the current work would not have an impact over and above other time factors induced by one's environment. On the other hand, the preparation and initiation of any well-learned skill may benefit from situations that limit the opportunity to explicitly attend to and monitor the activation and initiation of practiced motor programs best left outside conscious control. Indeed, Johnson and Raab's (2003) demonstration of the optimality of early decisions to act in team handball suggests that even in nonself-paced tasks, speed may have its benefits. Future research specifically addressing this issue will elucidate the boundary conditions of the speed advantage.

\section{Conclusions}

The current work examined the impact of time constraints on performance as a function of skill level and as a function of individuals' familiarity with the tools available to execute their skill. It was found that when using standard tools of the trade (i.e., a regular putter), novices performed at a higher level when they had more time available to both prepare for and execute their performance. Skilled golfers, on the other hand, show the opposite effect. In this case, however, this relation between putting time and putting outcome only held for the preparation component of per- formance. When skilled golfers were given an oddly shaped and weighted funny putter with which to execute their putts, skilled individuals looked more like novices until they were able to adapt to the novel constraints of the new tool. When performing a well-learned skill with a familiar implement, speeded performance has its benefits. But until one reaches such a stage or when faced with novel task demands, devoting what attentional resources and time one can to the step-by-step planning and unfolding of execution seems optimal.

\section{References}

Anderson, J. R. (1982). Acquisition of a cognitive skill. Psychological Review, 89, 369-406.

Anderson, J. R. (1983). The architecture of cognition. Cambridge, MA: Harvard University.

Anderson, J. R. (1993). Rules of mind. Hillsdale, NJ: Erlbaum.

Baumeister, R. F. (1984). Choking under pressure: Self-consciousness and paradoxical effects of incentives on skillful performance. Journal of Personality and Social Psychology, 46, 610-620.

Beilock, S. L., Bertenthal, B. I., McCoy, A. M., \& Carr, T. H. (2004). Haste does not always make waste: Expertise, direction of attention, and speed versus accuracy in performing sensorimotor skills. Psychonomic Bulletin \& Review, 11, 373-379.

Beilock, S. L., \& Carr, T. H. (2001). On the fragility of skilled performance: What governs choking under pressure? Journal of Experimental Psychology: General, 130, 701-725.

Beilock, S. L., \& Carr, T. H. (2005). When high-powered people fail: Working memory and "choking under pressure" in math. Psychological Science, 16, 101-105.

Beilock, S. L., Carr, T. H., MacMahon, C., \& Starkes, J. L. (2002). When paying attention becomes counterproductive: Impact of divided versus skill-focused attention on novice and experienced performance of sensorimotor skills. Journal of Experimental Psychology: Applied, 8, 6-16.

Beilock, S. L., \& Gonso, S. (2008). Putting in the mind vs. putting on the green: Expertise, performance time, and the linking of imagery and action. The Quarterly Journal of Experimental Psychology: Human Experimental Psychology, 61, 920-932.

Beilock, S. L., Jellison, W. A., Rydell, R. J., McConnell, A. R., \& Carr, T. H. (2006). On the causal mechanisms of stereotype threat: Can skills that don't rely heavily on working memory still be threatened? Personality \& Social Psychology Bulletin, 32, 1059-1071.

Beilock, S. L., Kulp, C. A., Holt, L. E., \& Carr, T. H. (2004). More on the fragility of performance: Choking under pressure in mathematical problem solving. Journal of Experimental Psychology: General, 133, 584-600.

Beilock, S. L., Wierenga, S. A., \& Carr, T. H. (2002). Expertise, attention, and memory in sensorimotor skill execution: Impact of novel task constraints on dual-task performance and episodic memory. The Quarterly Journal of Experimental Psychology: Human Experimental Psychology, 55, 1211-1240.

Brown, T. L., \& Carr, T. H. (1989). Automaticity in skill acquisition: Mechanisms for reducing interference in concurrent performance. Journal of Experimental Psychology: Human Perception and Performance, 15, 686-700

Bryan, W. L., \& Harter, N. (1899). Studies on the telegraphic language: The acquisition of a hierarchy of habits. Psychological Review, 6, 345-375.

Castaneda, B., \& Gray, R. (2007). Effects of focus of attention on baseball batting performance in players of differing skill levels. Journal of Sport \& Exercise Psychology, 29, 59-76.

Chein, J. M., Schneider, W. (2005). Neuroimaging studies of practicerelated change: FMRI and meta-analytic evidence of a domain-general control network for learning. Cognitive Brain Research, 25, 607-623.

Chi, M. T., Feltovitch, P. J., \& Glaser, R. (1981). Categorization and 
representation of physics problems by experts and novices. Cognitive Science, 5, 121-152.

Deeny, S. P., Hillman, C. H., Janelle, C. M., \& Hatfield, B. D. (2003). Cortico-cortical communication and superior performance in skilled marksmen: An EEG coherence Analysis. Journal of Sport \& Exercise Psychology, 25, 188-204.

Ericsson, K. A., \& Charness, N. (1994). Expert performance - Its structure and acquisition. American Psychologist, 49, 725-747.

Fitts, P. M. (1954). The information capacity of the human motor system in controlling the amplitude of movement. Journal of Experimental Psychology, 47, 381-391.

Fitts, P. M., \& Posner, M. I. (1967). Human performance. Belmont, CA: Brooks/Cole.

Ford, P., Hodges, N. J., \& Williams, A. M. (2005). Online attentional focus manipulations in a soccer-dribbling task: Implications for the proceduralization of motor skills. Journal of Motor Behavior, 37, 386-394.

Glover, S., \& Dixon, P. (2002). Semantics affect the planning but not control of grasping. Experimental Brain Research, 146, 383-387.

Glover, S., Rosenbaum, D. A., Graham, J., \& Dixon, P. (2004). Grasping the meaning of words. Experimental Brain Research, 154, 103-108.

Gray, R. (2004). Attending to the execution of a complex sensorimotor skill: Expertise differences, choking and slumps. Journal of Experimental Psychology: Applied, 10, 42-54.

Hatfield, B. D., \& Hillman, C. H. (2001). The psychophysiology of sport: A mechanistic understanding of the psychology of superior performance. In R. Singer, H. Hausenblas, \& C. Janelle (Eds.), Handbook of sport psychology (pp. 362-386). New York, NY: Wiley.

Jackson, R. C., Ashford, K. J., \& Norsworthy, G. (2006). Attentional focus, dispositional reinvestment and skilled motor performance under pressure. Journal of Sport \& Exercise Psychology, 28, 49-68.

Jancke, L., Shah, N. J., \& Peters, M. (2000). Cortical activation sin primary and secondary motor areas for complex bimanual movements in professional pianists. Cognitive Brain Research, 10, 177-183.

Janelle, C. M., Hillman, C. H., Apparies, R. J., Murray, N. P., Meili, L., Fallon, E. A., et al. (2000). Expertise differences in cortical activation and gaze behavior during rifle shooting. Journal of Sport \& Exercise Psychology, 22, 167-182.

Johnson, J. G., \& Raab, M. (2003). Take the first: Option-generation and resulting choices. Organizational Behavior and Human Decision Processes, 91, 215-229.

Kanfer, R., \& Ackerman, P. L. (1989). Motivation and cognitive abilities: An integrative/aptitude - treatment interaction approach to skill acquisition. Journal of Applied Psychology (Monograph), 74, 657-690.

Keele, S. W. (1986). Motor control. In K. R. Boff, L. Kaufman, \& J. P. Thomas (Eds.), Handbook of perception and human performance (Vol. 7). New York, NY: Wiley.

Keele, S. W., \& Summers, J. J. (1976). The structure of motor programs. In G. E. Stelmach (Ed.), Motor control: Issues and trends (pp. 109142). New York, NY: Academic Press.

Klein, G., Wolf, S., Militello, L., \& Zsambok, C. (2005). Characteristics of skilled option generation in chess. Organizational Behavior and Human Decision Processes, 62, 63-39.
Kruger, J., \& Dunning, D. (1999). Unskilled and unaware of it: How difficulties in recognizing one's own incompetence lead to inflated self-assessments. Journal of Personality and Social Psychology, 77, $1121-1134$

Lewis, B., \& Linder, D. (1997). Thinking about choking? Attentional processes and paradoxical performance. Personality and Social Psychology Bulletin, 23, 937-944.

MacKay, D. G. (1982). The problems of flexibility, fluency, and speedaccuracy trade-off in skilled behavior. Psychological Review, 89, 483-506.

Masters, R. S. W. (1992). Knowledge, knerves and know-how: The role of explicit versus implicit knowledge in the breakdown of a complex motor skill under pressure. British Journal of Psychology, 83, 343-358.

Masters, R. S. W. (2000). Theoretical aspects of implicit learning in sport. International Journal of Sport Psychology, 31, 530-541.

Milton, J., Solodkin, A., Hlustik, P., \& Small, S. L. (2007). The mind of expert motor performance is cool and focused. NeuroImage, 35, 804-813.

National Basketball Association (2006). NBA introduces new game ball. Retrieved February 4, 2008, from http://www.nba.com/news/ blackbox_060628.html

Perkins-Ceccato, N., Passmore, S. R., \& Lee, T. D. (2003). Effects of focus of attention depend on golfers' skill. Journal of Sports Sciences, 21, 593-600.

Poolton, J. M., Masters, R. S. W., Maxwell, J. P. (2005). The relationship between initial errorless learning conditions and subsequent performance. Human Movement Science, 24, 362-378.

Posner, M. I., \& Snyder, C. R. R. (1975). Attention and cognitive control In R. L. Solso (Ed.), Information processing and cognition: Loyola Symposium. Hillsdale, NJ: Erlbaum.

Proctor, R. W., \& Dutta, A. (1995). Skill acquisition and human performance. Thousand Oaks, CA: Sage.

Raab, M., \& Johnson, J. G. (2007). Expertise-based differences in search and option-generation strategies. Journal of Experimental Psychology: Applied, 13, 158-170.

Raichle, M. E., Fiez, J. A., Videen, T. O., Macleod, A. M. K., Pardo, J. V., Fox, P. T., et al. (1994). Practice-related changes in human brain functionalanatomy during nonmotor learning. Cerebral Cortex, 4, 8-26.

Schmidt, R. A., \& Lee, T. D. (2005). Motor control and learning: A behavioral emphasis. Champaign, IL: Human Kinetics.

Shiffrin, R. M., \& Schneider, W. (1977). Controlled and automatic human information processing: II. Perceptual learning, automatic attending, and a general theory. Psychological Review, 84, 127-190.

Vickers, J. N. (1996). Visual control while aiming at a far target. Journal of Experimental Psychology: Human Perception and Performance, 22, 342-354.

Woodworth, R. S. (1899). The accuracy of voluntary movement. Psychological Review Monograph, 3.

Received July 27, 2007

Revision received April 30, 2008

Accepted May 5, 2008 
